\title{
Towards a non-perturbative matching of HQET and QCD with dynamical light quarks*
}

\section{$\bar{F} L P H A$ \\ Collaboration}

\section{Michele Della Morte}

CERN, Physics Department, TH Unit, CH-1211 Geneva 23, Switzerland

E-mail: dellamor@mail.cern.ch

\section{Patrick Fritzsch, Jochen Heitger}

Westfälische Wilhelms-Universität Münster, Institut für Theoretische Physik,

Wilhelm-Klemm-Strasse 9, D-48149 Münster, Germany

E-mail: fritzsch@uni-muenster.de, heitger@uni-muenster.de

\section{Harvey B. Meyer}

Massachusets Institute of Technology, Center for Theoretical Physics,

Cambridge, MA 02139, U.S.A.

E-mail: meyerh@mit.edu

\section{Hubert Simma ${ }^{\dagger}$, Rainer Sommer}

Deutsches Elektronen-Synchrotron DESY, Zeuthen,

Platanenallee 6, D-15738 Zeuthen, Germany

E-mail: hubert.simma@desy.de, rainer.sommer@desy.de

We explain how the strategy of solving renormalization problems in HQET non-perturbatively by a matching to QCD in finite volume can be implemented to include dynamical fermions. As a primary application, some elements of an HQET computation of the mass of the b-quark beyond the leading order with $N_{\mathrm{f}}=2$ are outlined. In particular, the matching of HQET and QCD requires relativistic QCD simulations in a volume with $L \approx 0.5 \mathrm{fm}$, which will serve to quantitatively determine the heavy quark mass dependence of heavy-light meson observables in the continuum limit of finite-volume two-flavour lattice QCD. As a preparation for the latter, we report on our determination of the renormalization constants and improvement coefficients relating the renormalized current and subtracted bare quark mass in the relevant weak coupling region. The calculation of these coefficients employs a constant physics condition in the Schrödinger functional scheme, where the box size $L$ is fixed by working at a prescribed value of the renormalized coupling.

The XXV International Symposium on Lattice Field Theory

July 30 - August 42007

Regensburg, Germany

\footnotetext{
* Based on the contributions of J. Heitger and P. Fritzsch.

$\dagger$ Present address: Università di Milano "Bicocca”, Dip. di Fisica, Piazza della Scienza 3, I-20126 Milano, Italy
} 


\section{Introduction}

In the light of the expected progress in flavour physics thanks to the impending B-physics experiments [1,2], precision lattice QCD more and more becomes to play a crucial rôle for a quantitative and accurate interpretation of these experimental results in the framework of the Standard Model and beyond, since it provides a theoretically sound approach to non-perturbatively compute the contributing matrix elements of operators among hadronic states.

A particular problem of dealing with heavy-light systems involving the b-quark as the heavy flavour by means of lattice QCD consists in the two disparate intrinsic scales that actually accompany any lattice calculation: the lattice spacing, $a$, has to be much smaller than $1 / m_{\mathrm{b}}$ in order to allow for a fine enough resolution of the B-meson states in question, and the linear extent of the lattice volume, $L$, has to be large enough for finite-size effects to be under control. Heavy Quark Effective Theory (HQET) on the lattice [3, 4], however, which relies upon a systematic expansion of the QCD action and correlation functions in inverse powers of the heavy quark mass $(m)$ around the static limit, offers a formally reliable solution to this problem. Still, for lattice HQET and its numerical applications to lead to precise results with controlled systematic errors in practice, two shortcomings had to be left behind first.

One is the exponential growth of the noise-to-signal ratio in static-light correlation functions, which is a consequence of the appearance of power divergences in the effective theory. As demonstrated in studies in the quenched approximation [5-9] as well as in the theory with $N_{\mathrm{f}}=2 \mathrm{dy}$ namical quarks [10], this problem can be overcome by a clever modification of the traditional Eichten-Hill discretization of the static action.

Another difficulty, more serious on the theoretical level, is associated with the aforementioned power divergences. Since in the effective theory mixings among operators of different dimensions are present, already the static limit of HQET is affected by a power-law divergent $\left(\sim g_{0}^{2} / a\right)$ additive mass renormalization. Unless the theory is renormalized non-perturbatively [11], it follows from this power-law divergence of lowest-order HQET — and, of course, from further ones $\sim g_{0}^{2} / a^{n+1}$ that arise at $\mathrm{O}\left(1 / \mathrm{m}^{n}\right), n \geq 1$ - that the continuum limit does not exist owing to a remainder, which, at any finite order [12-14] in perturbation theory, diverges in the continuum limit.

In ref. [15] a general solution to the latter has been worked out and numerically implemented for a determination of the b-quark's mass in the static and quenched approximations as a test case. The method is based on a non-perturbative matching of HQET and QCD in finite volume. It was subsequently extended to also include the $\mathrm{O}(1 / \mathrm{m})$ terms into the quenched computations of the mass of the b-quark, $\bar{m}_{\mathrm{b}} \overline{\mathrm{MS}}\left(\bar{m}_{\mathrm{b}}\right)$ [16] (see refs. [17, 18] for recent reviews in broader context), and of the B-meson decay constant [19].

An attractive property of the strategy, briefly summarized in Section 2, is that most parts of the actual calculation do not involve very large lattices. Hence, it is natural to remove the quenched approximation as the dominating remaining systematic uncertainty in our previous works using this method. The additional computational effort required if dynamical quarks are included is only moderate, except for the last step that involves the extraction of B-meson properties from simulations in physically large volumes (with spatial extents of $\approx 2 \mathrm{fm}$ or more) and thus will be computationally much more demanding than the finite-volume simulations for the non-perturbative renormalization part. 
In the present report we outline the various steps towards an HQET computation of the mass of the b-quark including the $\mathrm{O}(1 / \mathrm{m})$ correction along the lines of refs. [15, 16] in two-flavour lattice QCD, where most of the emphasis is put on the renormalization of the effective theory through the non-perturbative matching to QCD in finite volume in order to perform the power-divergent subtractions. This step requires, in particular, a determination of the relation between the renormalization group invariant (RGI) and the subtracted bare heavy quark mass in the relevant parameter region of $N_{\mathrm{f}}=2 \mathrm{QCD}$, which we present together with numerical results on the corresponding renormalization constant and improvement coefficients in some detail in Section 3. Results from the matching itself, which has just been started at the time of writing, as well as from the necessary simulations of the effective theory in small and intermediate volumes will only be available at later stages of our project.

\section{Survey of the computational steps}

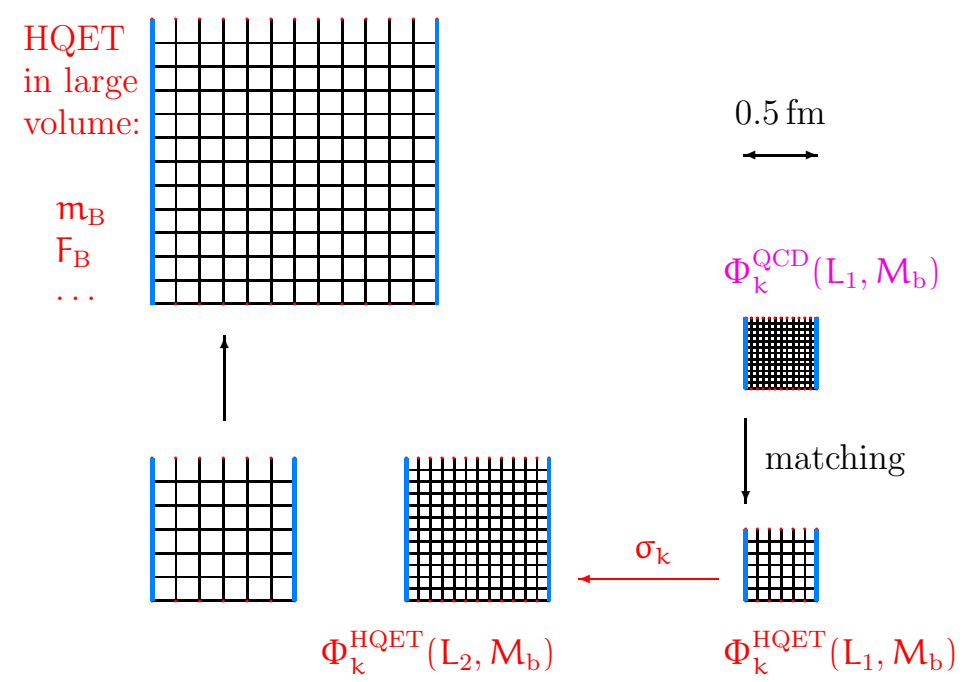

Figure 1: The strategy for performing computations in lattice HQET via a non-perturbative determination of the HQET parameters from QCD simulations in a small volume. It is designed such that steps indicated by arrows are to be repeated at smaller lattice spacings to reach a continuum limit.

Let us briefly recall the general strategy, introduced in [15]. It allows for a formulation of (zero-velocity) HQET in the framework of lattice QCD, where all steps of the computations including the renormalization are carried out non-perturbatively and the continuum limit can be taken.

The basic idea is illustrated in figure 1 and starts from a finite volume of extent $L_{1} \approx 0.5 \mathrm{fm}$. There, one chooses lattice spacings $a$ sufficiently smaller than $1 / m_{\mathrm{b}}$ such that the b-quark propagates correctly up to controllable discretization errors of order $a^{2}$. Since the relation between the RGI mass and the bare mass in QCD is known [20], suitable finite-volume observables $\Phi_{k}\left(L_{1}, M_{\mathrm{b}}\right)$ can be calculated as a function of the RGI b-quark mass, $M_{\mathrm{b}}$, and extrapolated to the continuum limit. The next step is to perform the power-divergent subtractions non-perturbatively by a set of matching conditions, in which the results obtained for $\Phi_{k}$ are equated to their representation in HQET (r.h.s. of figure 1). At the same physical value of $L_{1}$ but for resolutions $L_{1} / a=\mathrm{O}(10)$, the 
previously computed heavy-quark mass dependence of $\Phi_{k}\left(L_{1}, M_{\mathrm{b}}\right)$ in finite-volume QCD may be exploited to determine the bare parameters of the effective theory for $a \approx(0.025-0.05) \mathrm{fm}$. In order to evolve the HQET observables to large volumes, where contact with experiments can be made, one also computes them at these lattice spacings in a larger volume, $L_{2}=2 L_{1}$. The resulting relation between $\Phi_{k}\left(L_{1}\right)$ and $\Phi_{k}\left(L_{2}\right)$ is encoded in associated step scaling functions $\sigma_{k}$, as indicated in figure 1 as well. Finally, the knowledge of $\Phi_{k}\left(L_{2}, M_{\mathrm{b}}\right)$ and employing resolutions $L_{2} / a=\mathrm{O}(10)$ fixes the bare parameters of the effective theory for $a \approx(0.05-0.1) \mathrm{fm}$ so that a connection to those lattice spacings is established, where large-volume observables, such as the B-meson mass or decay constant, can be calculated (1.h.s. of figure 1).

Having in mind the computation of $M_{\mathrm{b}}$ as the specific application, this sequence of steps yields an expression of $m_{\mathrm{B}}$ (taken to be the physical input) as a function of $M_{\mathrm{b}}$ via the quark mass dependence of $\Phi_{k}\left(L_{1}, M_{\mathrm{b}}\right)$, which eventually can be inverted to arrive at the desired physical value of the RGI b-quark mass extracted from the effective theory. As pointed out before, the whole construction is such that its various pieces separately have a continuum limit. With the realization of this strategy for the quenched case it was shown in ref. [16] that a determination of $M_{\mathrm{b}}$ including $\mathrm{O}(1 / m)$ in HQET only requires up to three matching observables, $\Phi_{1}, \Phi_{2}$ and $\Phi_{3}$, if the spin-averaged B-meson mass is used as physical input. That is also the path we will follow in our present extension to the case of two-flavour QCD.

\subsection{Definition of the matching volume}

We consider QCD with $N_{\mathrm{f}}=2$ mass-degenerate dynamical quarks, which are identified with up and down. All other quarks are treated in the quenched approximation. A particularly convenient renormalization scheme, in which finite-volume observables suitable for a non-perturbative matching of the effective theory with QCD can readily be constructed [15, 21, 22], is the Schrödinger functional (SF) [23]. Relativistic and static quarks were introduced in [24] and [25], respectively, where in the latter reference it was found that the HQET expansion of the boundary quark fields is trivial up to and including $1 / m$-terms. ${ }^{1}$ Adopting any unexplained notation from refs. [15, 25], we only mention the periodicity phase $\theta$ of the fermion fields as a further kinematic parameter and the fact that homogeneous Dirichlet boundary conditions in time at $x_{0}=0$ and $x_{0}=T$ are employed. Since the parameters $\theta$ and masses of the quenched quarks can be set independently of those of the sea quarks, the basic situation for extracting heavy-light physics from SF correlation functions is the same as in the quenched approximation $[15,16]$. Moreover, in the finite-volume simulations we set $\theta=0.5$ for the dynamical light quarks and their PCAC mass to zero, $m_{1}=0$.

The quantities $\Phi_{k}$ that enter the non-perturbative matching procedure described above have to be evaluated in the continuum limit. To this end we want to compute them for a series of bare parameters $\left(L / a, \beta, \kappa_{1}\right)$ such that the renormalized parameters in the light quark sector are fixed and thereby physics is kept constant along the approach to the continuum limit. Here, $\kappa_{1}$ denotes the hopping parameter of the dynamical light quarks. Our constant physics condition on the renormalized SF coupling, $\bar{g}^{2}(L)$, and the light quark mass reads

$$
\bar{g}^{2}\left(L_{0}\right)=2.989, \quad L_{0}=\frac{L_{1}}{2}, \quad L_{0} m_{1}\left(L_{0}\right)=0 .
$$

\footnotetext{
${ }^{1}$ From now on, $m$ generically denotes the mass parameter of the heavy quark treated in the effective theory, while the masses of the non-degenerate quark flavours in the relativistic theory are distinguished explicitly where necessary.
} 
This choice now defines the spatial extent $L_{1}$ of the volume, in which the matching between HQET and QCD is performed. Although an exact knowledge of $L_{1}$ in physical units in not yet needed at this stage, one can already infer from the known running of the SF coupling for $N_{\mathrm{f}}=2$ [26] that $L_{1} \approx 0.5 \mathrm{fm}$. Hence, we will finally have $L_{2}=2 L_{1} \approx 1 \mathrm{fm}$ and thus $L_{\infty} \equiv 4 L_{1} \approx 2 \mathrm{fm}$ for the large volume, which is well consistent with the envisaged strategy, figure 1 .

We have fixed $\bar{g}^{2}\left(L_{1} / 2\right)=2.989$ by a new simulation at $L_{0} / a=20, T=L_{0}$, and made tentative interpolations in $\beta=6 / g_{0}^{2}$ for given $L_{0} / a \leq 16$ to this target value, based on the known dependence of the SF coupling and the current quark mass on the bare parameters $(\beta, \kappa)$ available from the data of ref. [26]. Using the known $\beta$-function and our experience from the quenched calculation [16], we can estimate that an uncertainty of about 0.04 in the coupling will translate via the resulting one in $L_{1}$ into an uncertainty in the b-quark mass of at most $0.5 \%$. The condition of zero light quark mass in eq. (2.1) is met by setting $\kappa \equiv \kappa_{1}$ to the critical hopping parameter, $\kappa_{\mathrm{c}}$, estimated again on basis of published data [26], whereby a slight mismatch of $\left|L_{0} m_{1}\left(L_{0}\right)\right|<0.05$ of this condition is tolerable in practice. The triples $\left(L_{0} / a, \beta, \kappa_{1}\right)$, which approximately define the extent $L_{1}$ of the matching volume through eq. (2.1) and which are used in our subsequent study of improvement and renormalization factors, are collected in columns $2-4$ of table 1 in Section 3.

The preliminary interpolation procedure for $\bar{g}^{2}(\beta)$ underlying these $\beta$-values is currently being checked (and refined) by direct simulations, in order to avoid a non-negligible systematic error from small violations of the condition (2.1) on the final results. Yet, this will affect our estimates of $b_{\mathrm{m}}$ and $Z$ in Section 3 only at a negligible level, because there any deviation from the line of constant physics only entails a small change of the $\mathrm{O}\left(a^{2}\right)$ effects.

\subsection{Fixing the heavy quark mass in finite-volume QCD}

Having fixed $L_{1}$ via enforcing constant physics at $L_{0}=L_{1} / 2$, the computation of the heavy quark mass dependence of the finite-volume observables $\Phi_{k}$, which is the key element in the nonperturbative matching step within our strategy, will amount to evaluate heavy-light SF correlation functions in a volume $L_{1}^{3} \times T, T=L_{1}$, for a series of precisely fixed values of the renormalized heavy quark mass covering the b-quark mass region.

This is achieved by exploiting the $\mathrm{O}(a)$ improved relation between the (subtracted) bare heavy quark mass $m_{\mathrm{q}, \mathrm{h}}$ and the RGI mass [20, 27], viz.

$$
M=h\left(L_{0}\right) Z_{\mathrm{m}}\left(g_{0}, L_{0} / a\right) m_{\mathrm{q}, \mathrm{h}}\left(1+b_{\mathrm{m}}\left(g_{0}\right) a m_{\mathrm{q}, \mathrm{h}}\right)+\mathrm{O}\left(a^{2}\right),
$$

where

$$
Z_{\mathrm{m}}\left(g_{0}, L_{0} / a\right)=\frac{Z\left(g_{0}\right) Z_{\mathrm{A}}\left(g_{0}\right)}{Z_{\mathrm{P}}\left(g_{0}, L_{0} / a\right)}, \quad a m_{\mathrm{q}, \mathrm{h}}=\frac{1}{2}\left(\frac{1}{\kappa_{\mathrm{h}}}-\frac{1}{\kappa_{\mathrm{c}}}\right)
$$

and $Z_{\mathrm{A}}$ is known non-perturbatively from ref. [28]. The scale dependent renormalization constant $Z_{\mathrm{P}}$ may be calculated for the relevant couplings on $L_{0}^{3} \times T$ lattices with $T=L_{0}$ in the same way as in ref. [20]. The factor

$$
h\left(L_{0}\right)=\frac{M}{\bar{m}\left(\mu_{0}\right)}=1.521(14), \quad \mu_{0}=\frac{1}{L_{0}}=\frac{2}{L_{1}},
$$

represents the universal, regularization independent ratio of the RGI heavy quark mass, $M$, to the running quark mass $\bar{m}$ in the SF scheme at the renormalization scale $\mu_{0} . h\left(L_{0}\right)$ was evaluated by a reanalysis of the $N_{\mathrm{f}}=2$ non-perturbative quark mass renormalization data published in ref. [20]. 
Therefore, in order to specify the hopping parameters of the heavy flavour, $\kappa_{\mathrm{h}}$, which according to eq. (2.2) for given $L_{1} / a=2 L_{0} / a$ and $\beta=6 / g_{0}^{2}$ correspond to a series of dimensionless RGI quark mass values $z \equiv L_{1} M$ in the b-quark region, it remains to accurately determine the improvement coefficient $b_{\mathrm{m}}$ and the renormalization constant $Z$. We discuss this computation for the relevant weak coupling range (cf. table 1) of $\mathrm{O}(a)$ improved two-flavour lattice QCD in the next section.

\subsection{Preparing for the finite-volume computations}

\subsubsection{Matching to QCD}

As mentioned in the foregoing subsection, on the QCD side this step consists in calculating the quark mass dependence of the quantities $\Phi_{1}, \Phi_{2}$ and $\Phi_{3}$ in the volume $L_{1}^{4}$. For the exact definitions of these effective heavy-light meson energies in terms of SF correlators we refer to ref. [16]. In addition to $L_{1}^{3} \times T, T=L_{1}$, lattices with $T=L_{1} / 2$ will also be needed (cf. Appendix C of [16]).

The aforesaid fine-tuning of $\beta$ for $L_{1} /(2 a) \leq 16$ to satisfy the condition $\bar{g}^{2}\left(L_{1} / 2\right)=2.989$, eq. (2.1), with a precision $\Delta \bar{g}^{2} \lesssim 0.04$ requires up to $\left(L_{1} / a\right)^{4}=\left(2 L_{0} / a\right)^{4}=40^{4}$ lattices with $N_{\mathrm{f}}=2$ at sea quark parameters close to those quoted in table 1 in order to reach the continuum limit.

\subsubsection{Parameters for HQET simulations in $L_{1}^{3} \times T$ with $T=L_{1}, L_{1} / 2$}

For the determination of the step scaling functions $L_{1} \rightarrow L_{2}=2 L_{1}$ belonging to the $\Phi_{k}$ 's counterparts in HQET, we must fix the simulation parameters for resolutions $6 \leq L_{1} / a \leq 16$. The corresponding constraint on the renormalized coupling at $L_{1}$ is $\bar{g}^{2}\left(L_{1}\right)=\sigma(2.989)=4.484(48)$ [26].

As a starting point for the tuning of $\bar{g}^{2}\left(L_{1}\right)$ at each $L_{1} / a$, we introduce another low-energy scale, $L^{*}$, defined via $\bar{g}^{2}\left(L^{*}\right)=5.5$ and obeying [29]

$$
\ln \left(L^{*} / a\right)=2.3338+1.4025(\beta-5.5), \quad \beta \in[5.3,5.8], \quad L^{*} / a \in[7.8,16.1],
$$

which allows to estimate the ratio $r_{1}=L_{1} / L^{*} \approx 0.8$ in the continuum limit. Trial $\beta$-estimates for the range of $L_{1} / a$ in question are then obtained from the parameterization (2.5) and improved by further simulations, aiming at a precision of $\Delta \bar{g}^{2} \lesssim 0.1$. This will be finished soon.

Small mismatches of the simulation results w.r.t. the target values, i.e. $\bar{g}^{2}\left(L_{1}\right)=4.484$ and $L_{1} m_{1}\left(L_{1}\right)=0$, may be corrected by the non-perturbative $\beta$-function and the mass derivative of the coupling $[26,29]$.

\subsubsection{Parameters for HQET simulations in $L_{2}^{3} \times T$ with $T=L_{2}, L_{2} / 2$}

To prepare for the power-divergent subtractions in the volume of extent $L_{2}=2 L_{1} \approx 1 \mathrm{fm}$ within the effective theory that eventually provide the link to HQET observables in the physically large volume (of extent $L_{\infty}$ ), the two-flavour theory will have to be simulated at typical resolutions of about $L_{2} / a=8,12,16$ and lattice spacings corresponding to $5.3 \lesssim \beta \lesssim 5.9$.

For fixing the necessary simulation parameters by means of the condition of fixed coupling $\bar{g}^{2}\left(L_{2}\right)$, one can rely again on the scale $L^{*}$ and its ratio to $L_{2}, r_{2}=L_{2} /\left.L^{*}\right|_{\text {continuum }} \approx 1.6$, and infer the wanted pairs $\left(L_{2} / a, \beta\right)$ from eq. (2.5). 


\section{Computation of the missing improvement and renormalization factors}

We now present our non-perturbative determination of the improvement coefficient $b_{\mathrm{m}}$ and the renormalization constant $Z$ in the $\beta$-range relevant for the matching of HQET to QCD in small volume, such that the RGI heavy quark mass can be set to desired values $z=L_{1} M$.

Our generation of unquenched gauge configurations with SF boundary conditions for $N_{\mathrm{f}}=2$ $\mathrm{O}(a)$ improved massless Wilson quarks employs the hybrid Monte Carlo (HMC) algorithm [30] in its variant used in the study of autocorrelation times in ref. [31]. It comprises multi-time-scale integration schemes $[32,33]$ with mass preconditioning [34-36] on top of even-odd preconditioning.

\subsection{Non-degenerate current quark masses and estimators for $b_{\mathrm{A}}-b_{\mathrm{P}}, b_{\mathrm{m}}$ and $Z$}

We proceed following the idea of imposing improvement conditions at constant physics, which was first advocated in [27] and already applied to the present situation but for $N_{\mathrm{f}}=0$ in [21].

Since the definition (2.1) of $L_{1}$ via the renormalized coupling $\bar{g}^{2}\left(L_{1} / 2\right)=\bar{g}^{2}\left(L_{0}\right)$, respectively the bare parameters in columns $2-4$ of table 1 complying with it, have such a constant physics condition built in from the start, we can directly work at those pairs of $\left(L_{0} / a, \beta\right)$. With this as our choice of improvement condition, supplemented by the SF-specific settings of zero boundary fields, $\theta=0.5$ and - just for the purpose of this section $-T / L_{0}=3 / 2$, the improvement coefficients $b_{\mathrm{A}}-b_{\mathrm{P}}$ and $b_{\mathrm{m}}$ and the renormalization constant $Z$ become smooth functions of $g_{0}^{2}$ in the region where they are needed. ${ }^{2}$

Taking over any unexplained notations and details from refs. [21, 27] (and references therein), $b_{\mathrm{A}}-b_{\mathrm{P}}, b_{\mathrm{m}}$ and $Z$ can be determined by studying $\mathrm{QCD}$ with non-degenerate valence quarks. Treating the latter in the quenched approximation, the structure of the $\mathrm{O}(a)$ improved theory in conjunction with a massless renormalization scheme retains the relative simplicity of the $N_{\mathrm{f}}=0$ case elaborated in ref. [27]. For instance, the improvement of the off-diagonal bilinear fields $X^{ \pm}=X^{1} \pm i X^{2}$, $X=A_{\mu}, P$, emerging as a consequence of the broken isospin symmetry in flavour space, is the same as in the degenerate case, except that the $b$-coefficients now multiply the average $\frac{1}{2}\left(a m_{\mathrm{q}, i}+a m_{\mathrm{q}, j}\right)$ of the subtracted bare quark masses, $m_{\mathrm{q}, i}=m_{0, i}-m_{\mathrm{c}}$, which themselves are separately improved for each quark flavour:

$$
\widetilde{m}_{\mathrm{q}, i}=m_{\mathrm{q}, i}\left(1+b_{\mathrm{m}} a m_{\mathrm{q}, i}\right) .
$$

(Here and below the indices $i, j$ label the different quark flavours.) Identifying the valence flavours in the isospin doublet with a light (strange) and a heavy (bottom) quark, the corresponding PCAC relation reads

$$
\partial_{\mu} A_{\mu}^{ \pm}(x)=\left(m_{i}+m_{j}\right) P^{ \pm}(x),
$$

and the renormalization constants $Z_{\mathrm{A}}$ and $Z_{\mathrm{P}}$ that come into play upon renormalization are just those known in the theory with two mass-degenerate quarks.

Accordingly, the SF correlation functions involving the axial current and the pseudoscalar density generalize to $f_{\mathrm{A}}^{i j}\left(x_{0}\right)=-\frac{1}{2}\left\langle A_{0}^{+}(x) O^{-}\right\rangle$and $f_{\mathrm{P}}^{i j}\left(x_{0}\right)=-\frac{1}{2}\left\langle P^{+}(x) O^{-}\right\rangle$, with pseudoscalar boundary sources decomposed as $O^{ \pm}=O^{1} \pm i O^{2}$ where $O^{a}=a^{6} \sum_{\mathbf{y}, \mathbf{z}} \bar{\zeta}(\mathbf{y}) \gamma_{5} \frac{1}{2} \tau^{a} \zeta(\mathbf{z})$. Then the

\footnotetext{
${ }^{2}$ Although the difference of coefficients $b_{\mathrm{A}}-b_{\mathrm{P}}$ is actually not needed for fixing the RGI mass through eq. (2.2), we include it in the present discussion.
} 
improved bare PCAC (current) quark masses ${ }^{3}$ as functions of the timeslice location $x_{0}$ are given by

$$
m_{i j}\left(x_{0} ; L_{0} / a, T / L_{0}, \theta\right)=\frac{\tilde{\partial}_{0} f_{\mathrm{A}}^{i j}\left(x_{0}\right)+a c_{\mathrm{A}} \partial_{0}^{*} \partial_{0} f_{\mathrm{P}}^{i j}\left(x_{0}\right)}{2 f_{\mathrm{P}}^{i j}\left(x_{0}\right)},
$$

where only here we explicitly indicate their additional dependence on $L_{0} / a, T / L_{0}$ and $\theta$. In the degenerate case, $i=j$, the correlators assume the standard form, and $m_{i j}$ just reduces to the current quark mass of a single quark flavour that is prepared by a corresponding choice of equal values for the associated hopping parameters, $\kappa_{i}=\kappa_{j}$. Also the precise definition of the lattice derivatives in eq. (3.3) matters. As it is written there, $\tilde{\partial}_{0}=\frac{1}{2}\left(\partial_{0}+\partial_{0}^{*}\right)$ denotes the average of the ordinary forward and backward derivatives, but as in refs. [21,27] we have used the improved derivatives

$$
\tilde{\partial}_{0} \rightarrow \tilde{\partial}_{0}\left(1-\frac{1}{6} a^{2} \partial_{0}^{*} \partial_{0}\right), \quad \partial_{0}^{*} \partial_{0} \rightarrow \partial_{0}^{*} \partial_{0}\left(1-\frac{1}{12} a^{2} \partial_{0}^{*} \partial_{0}\right)
$$

as well, which (when acting on smooth functions) have $\mathrm{O}\left(g_{0}^{2} a^{2}, a^{4}\right)$ errors only.

For their numerical calculation, the coefficients $b_{\mathrm{A}}-b_{\mathrm{P}}, b_{\mathrm{m}}$ and the finite factor $Z=Z_{\mathrm{m}} Z_{\mathrm{P}} / Z_{\mathrm{A}}$ (see eq. (2.3)) are isolated by virtue of the identity

$$
m_{i j}=Z\left[\frac{1}{2}\left(m_{\mathrm{q}, i}+m_{\mathrm{q}, j}\right)+\frac{1}{2} b_{\mathrm{m}}\left(a m_{\mathrm{q}, i}^{2}+a m_{\mathrm{q}, j}^{2}\right)-\frac{1}{4}\left(b_{\mathrm{A}}-b_{\mathrm{P}}\right) a\left(m_{\mathrm{q}, i}+m_{\mathrm{q}, j}\right)^{2}\right]+\mathrm{O}\left(a^{2}\right) .
$$

It is obtained by equating the expression for the $\mathrm{O}(a)$ improved renormalized quark mass in terms of the bare PCAC mass with the alternative expression in terms of the subtracted bare quark mass. Forming ratios of suitable combinations of degenerate and non-degenerate current quark masses in the representation (3.5) then enables to derive direct estimators for $b_{\mathrm{A}}-b_{\mathrm{P}}, b_{\mathrm{m}}$ and $Z$ [27]:

$$
\begin{gathered}
R_{\mathrm{AP}}=\frac{2\left(2 m_{12}-m_{11}-m_{22}\right)}{\left(m_{11}-m_{22}\right)\left(a m_{\mathrm{q}, 1}-a m_{\mathrm{q}, 2}\right)}=b_{\mathrm{A}}-b_{\mathrm{P}}+\mathrm{O}\left(a m_{\mathrm{q}, 1}+a m_{\mathrm{q}, 2}\right), \\
R_{\mathrm{m}}=\frac{4\left(m_{12}-m_{33}\right)}{\left(m_{11}-m_{22}\right)\left(a m_{\mathrm{q}, 1}-a m_{\mathrm{q}, 2}\right)}=b_{\mathrm{m}}+\mathrm{O}\left(a m_{\mathrm{q}, 1}+a m_{\mathrm{q}, 2}\right),
\end{gathered}
$$

with $m_{0,3}=\frac{1}{2}\left(m_{0,1}+m_{0,2}\right)$, neglecting other quark mass independent lattice artifacts of $\mathrm{O}(a)$. For the renormalization constant $Z$ an analogous formula holds even up to $\mathrm{O}\left(a^{2}\right)$ corrections,

$$
R_{Z}=\frac{m_{11}-m_{22}}{m_{\mathrm{q}, 1}-m_{\mathrm{q}, 2}}+\left(b_{\mathrm{A}}-b_{\mathrm{P}}-b_{\mathrm{m}}\right)\left(a m_{11}+a m_{22}\right)=Z+\mathrm{O}\left(a^{2}\right),
$$

if the correct value for $b_{\mathrm{A}}-b_{\mathrm{P}}-b_{\mathrm{m}}=R_{\mathrm{AP}}-R_{\mathrm{m}}$ (only involving correlation functions with mass degenerate quarks) is inserted. Note that generically the combination $Z=Z_{\mathrm{m}} Z_{\mathrm{P}} / Z_{\mathrm{A}}$ is a function of the improved bare coupling, $\tilde{g}_{0}^{2}=g_{0}^{2}\left(1+b_{\mathrm{g}} a m_{\mathrm{q}}\right)$. Since, however, we only consider light sea quarks that are massless (i.e. such that $m_{1} \approx 0$ ) and the valence quarks are anyway treated in the quenched approximation, this fact can be ignored here.

Still, to complete our definition of the line of constant physics, values for the bare PCAC masses of the valence quarks must be selected. As in [21], we consider two pairs,

$$
\begin{array}{ll}
\text { choice 1: } & L_{0} m_{11} \approx 0, \quad L_{0} m_{22} \approx 0.5, \\
\text { choice 2: } & L_{0} m_{11} \approx 0, \quad L_{0} m_{22} \approx 2.4 .
\end{array}
$$

${ }^{3}$ This expression for the PCAC masses is only $\mathrm{O}(a)$ improved up to a factor $1+\frac{1}{2}\left(b_{\mathrm{A}}-b_{\mathrm{P}}\right)\left(a m_{\mathrm{q}, i}+a m_{\mathrm{q}, j}\right)$ for quark mass dependent cutoff effects. 


\begin{tabular}{ccccccccc}
\hline set & $L_{0} / a$ & $\beta$ & $\kappa_{\mathrm{l}}$ & $\kappa_{\mathrm{h}}$ & $L_{0} m_{22}$ & $b_{\mathrm{A}}-b_{\mathrm{P}}$ & $b_{\mathrm{m}}$ & $Z$ \\
\hline 1 & 10 & 6.1906 & 0.136016 & 0.134318 & $0.4929(6)$ & $-0.0006(9)$ & $-0.6643(9)$ & $1.1046(1)$ \\
& 12 & 6.3158 & 0.135793 & 0.134378 & $0.4952(9)$ & $-0.003(2)$ & $-0.668(2)$ & $1.1050(2)$ \\
& 16 & 6.5113 & 0.135441 & 0.134387 & $0.492(1)$ & $-0.006(2)$ & $-0.667(3)$ & $1.1044(2)$ \\
& 20 & 6.6380 & 0.135163 & 0.134356 & $0.5005(9)$ & $-0.005(3)$ & $-0.669(3)$ & $1.1038(2)$ \\
\hline \multirow{2}{*}{2} & 10 & 6.1906 & 0.136016 & 0.127622 & $2.2909(5)$ & $+0.0727(4)$ & $-0.5655(3)$ & $1.0954(1)$ \\
& 12 & 6.3158 & 0.135793 & 0.128755 & $2.3475(7)$ & $+0.0513(5)$ & $-0.5785(5)$ & $1.0974(1)$ \\
& 16 & 6.5113 & 0.135441 & 0.130146 & $2.407(1)$ & $+0.0297(7)$ & $-0.5964(8)$ & $1.0995(1)$ \\
& 20 & 6.6380 & 0.135163 & 0.130965 & $2.4433(8)$ & $+0.0215(6)$ & $-0.6076(8)$ & $1.1002(1)$ \\
\hline
\end{tabular}

Table 1: Lattice parameters and numerical results on the improvement coefficients $b_{\mathrm{A}}-b_{\mathrm{P}}$ and $b_{\mathrm{m}}$ and on the renormalization constant $Z$. The parameters $\left(L_{0} / a, \beta, \kappa_{\mathrm{l}}\right)$ referring to the light (sea) quark sector have fixed SF coupling, $\bar{g}^{2}\left(L_{0}\right)=2.9(1)$, and vanishing quark mass such as to meet the constant physics condition of Section 2.1. Our results for $b_{\mathrm{A}}-b_{\mathrm{P}}, b_{\mathrm{m}}$ and $Z$ are based on statistics varying from $\mathrm{O}(300)$ measurements $\left(L_{0} / a=20\right)$ to $\mathrm{O}(2000)$ measurements $\left(L_{0} / a=10\right)$. The upper set refers to "choice 1 ", eq. (3.9), where the heavy quark mass is kept at $L_{0} m_{22} \approx 0.5$, while the lower set belongs to "choice 2 " with $L_{0} m_{22} \approx 2.4$, eq. (3.10). The condition $L_{0} m_{11} \approx 0$ is fulfilled up to negligible deviations of about 0.015 at most.

The first choice on $L_{0} m_{22}$ is motivated by the quenched investigation [27], where it was argued to be advantageous w.r.t. the size of $\mathrm{O}(a)$ ambiguities encountered, while with the second choice one is closer to the typical b-quark region itself. Satisfying these conditions on $L_{0} m_{22}$ for all $\left(L_{0} / a, \beta\right)$ in table 1 demands to properly adjust the hopping parameter, called $\kappa_{\mathrm{h}}$ above, that is responsible for the mass value of the heavy valence quark flavour. This in turn amounts to prior evaluations of the relevant correlation functions on the dynamical gauge background for some trial guesses of $\kappa_{\mathrm{h}}$, in order to estimate the heavy flavour's PCAC mass through eq. (3.3) and to tune it to the values dictated by eqs. (3.9) and (3.10) up to a few percent. ${ }^{4}$ The resulting hopping parameters are given in the fifth column of table 1 .

\subsection{Results}

The technical aspects of the analysis to compute the estimators (3.6) - (3.8) from the numerical data on the heavy-light SF correlation functions by means of the PCAC masses $m_{i j}$ for the various (degenerate and non-degenerate) valence quark mass combinations are the same as in refs. [21, 27]. The correlators have been evaluated on our dynamical gauge field configurations, which were generated on $L_{0}^{3} \times T$ lattices with $T=3 L_{0} / 2$ and massless sea quarks (thus complying with the above requirement $\kappa_{\mathrm{l}}=\kappa_{\mathrm{c}}$ resp. $L_{0} m_{11} \approx 0$ for the light valence quark flavour) and which were separated by $5-10 \mathrm{HMC}$ trajectories of length one. As for the $m_{i j}$ themselves, they have been calculated from the local masses, eq. (3.3), using improved derivatives (3.4) throughout and averaging over the central timeslices $L_{0} /(2 a), \ldots,\left(T-L_{0} / 2\right) / a$ to increase statistics. Being secondary quantities in particular, the statistical errors of the masses and of the $R_{\mathrm{X}}, \mathrm{X}=\mathrm{AP}, \mathrm{m}, Z$, obtained from them were estimated by the $\Gamma$-method [37], which directly analyzes autocorrelation functions.

\footnotetext{
${ }^{4}$ Similar to the situation in refs. [21, 27], this is to sufficient precision equivalent to keeping fixed the corresponding renormalized masses $L_{0} Z_{\mathrm{A}} m / Z_{\mathrm{P}}$, as for the considered couplings the entering renormalization constant barely varies.
} 


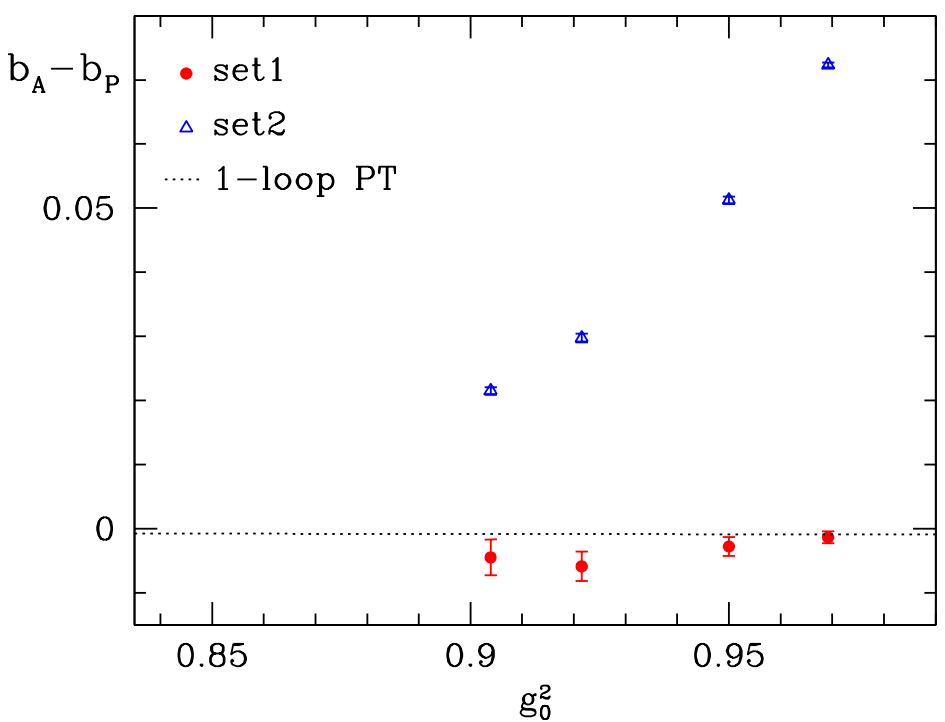

Figure 2: Two sets of non-perturbative results for $b_{\mathrm{A}}-b_{\mathrm{P}}$ in the considered region of bare couplings, referring to our two choices of quark masses, together with the prediction from one-loop perturbation theory.

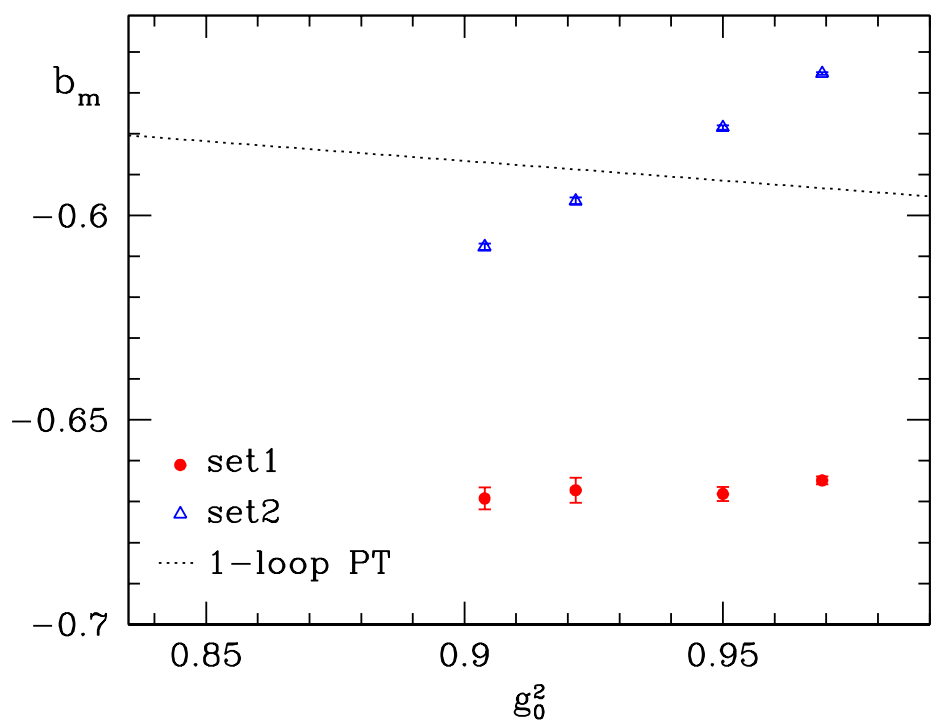

Figure 3: The same as in figure 2 but for the improvement coefficient $b_{\mathrm{m}}$.

Our non-perturbative results on $b_{\mathrm{A}}-b_{\mathrm{P}}, b_{\mathrm{m}}$ and $Z$ are also listed in table 1 . As a consequence of the underlying constant physics condition (2.1), the estimates $R_{\mathrm{X}}, \mathrm{X}=\mathrm{AP}, \mathrm{m}, Z$, become smooth functions of the bare coupling, $g_{0}^{2}=6 / \beta$. This is well reflected in figures $2-4$, where our results are shown in comparison with the one-loop perturbative predictions [27, 38].

The overall $g_{0}^{2}$-dependence of our results is qualitatively similar to the quenched study [21] and even comparable on the quantitative level. Whereas $R_{\mathrm{AP}}$ is compatible with a nearly vanishing $b_{\mathrm{A}}-b_{\mathrm{P}}$, as predicted by leading-order perturbation theory, for "choice 1" of quark masses and appears to approach this line quite rapidly as $g_{0}^{2} \rightarrow 0$ for "choice 2 ", one observes for both choices significant deviations of the sets of estimates for $b_{\mathrm{m}}$ and $Z$ from the leading perturbative behaviour 


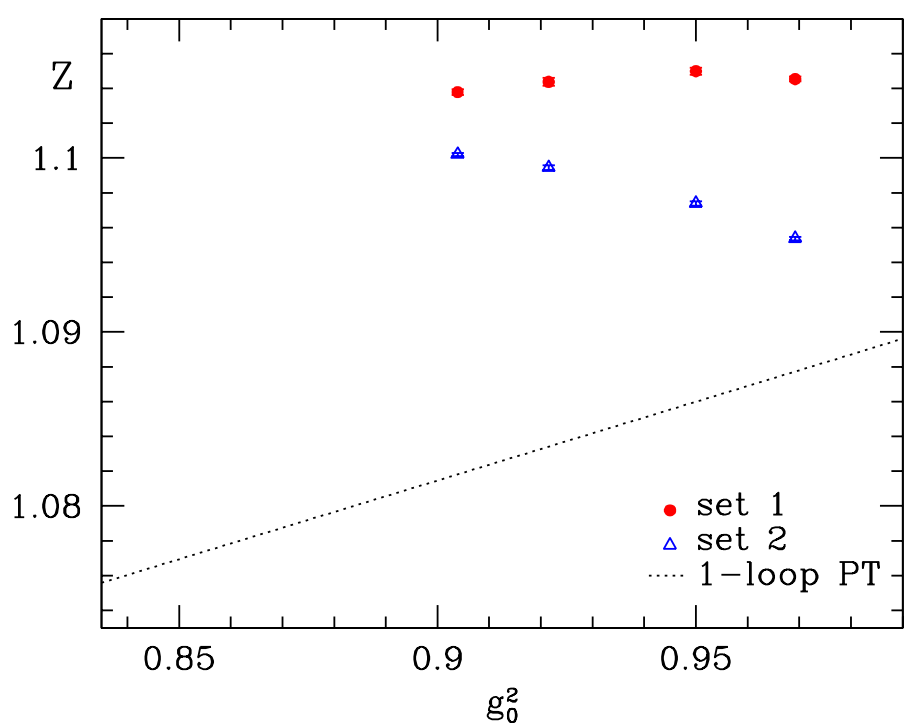

Figure 4: The same as in figure 2 but for the renormalization constant $Z$.
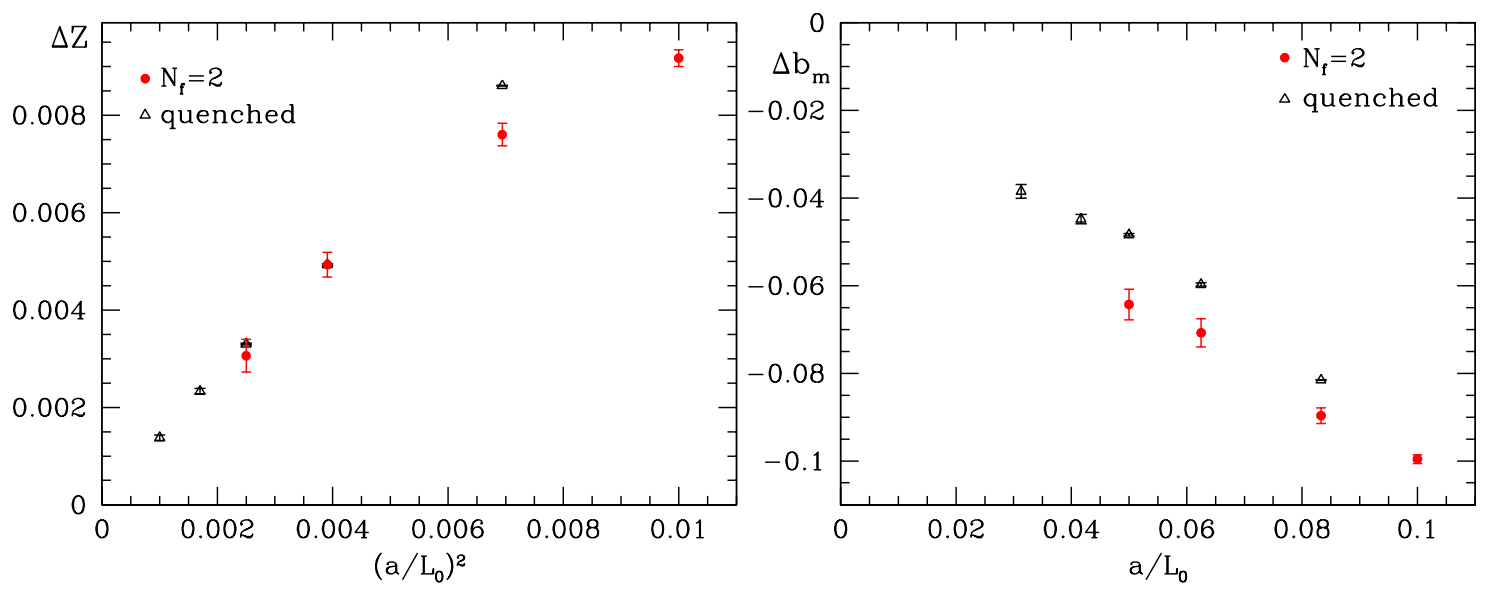

Figure 5: Left: Difference of the two sets of results in table 1 on the renormalization constant $Z$ versus $\left(a / L_{0}\right)^{2}$. Right: The same for the improvement coefficient $b_{\mathrm{m}}$ where, however, the ambiguity inherent in any improvement condition imposed is of $\mathrm{O}(a)$. The open black triangles display the corresponding quenched results from [21] for comparison.

in the weak coupling region considered. Since one expects the perturbative curves eventually to be approached in the limit $g_{0}^{2} \rightarrow 0$ also in case of $b_{\mathrm{m}}$ and $Z$, the curvature seen in our numbers hints at a more complicated structure of (unknown) higher-order terms. Hence, we have to conclude that if an improvement condition were used in a region of stronger couplings, which would no doubt lead to a rather different set of data points, simple one-loop perturbation theory would not be an adequate guide for the continuation of $b_{\mathrm{m}}$ and $Z$ to weak couplings. On the contrary, this would induce a source of uncertainty in results deriving from them that is difficult to control and, therefore, highlights the importance of employing improvement conditions in the $\beta$-range relevant to the actual application.

Of course, any other estimate $R_{\mathrm{X}}$ (i.e. stemming from a different choice of renormaliza- 
tion/improvement condition) may yield a different functional dependence upon $g_{0}^{2}$, but its differences are again smooth functions that vanish in the continuum limit with a rate proportional to $a / L_{0}$ (for improvement coefficients) or $\left(a / L_{0}\right)^{2}$ (for renormalization constants). These intrinsic $\mathrm{O}\left(a^{n}\right)$ ambiguities $(n=1,2)$ imply that rather than a numerical value at some given $\beta$, the important information lies in the correct $g_{0}^{2}$-dependence of the estimators $R_{\mathrm{X}}, \mathrm{X}=\mathrm{AP}, \mathrm{m}, Z$, obtained at constant physics. To demonstrate this, we also investigated a few alternative improvement conditions, which are either provided by defining the estimators $R_{\mathrm{X}}$ with standard instead of improved derivatives or by the two quark mass choices, eqs. (3.9) and (3.10), themselves. As an example we plot in the

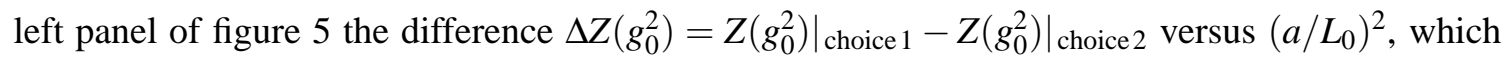
clearly exhibits a linear approach towards zero. Other cases behave similarly, e.g. the $\mathrm{O}(a)$ ambiguities for $\Delta b_{\mathrm{m}}\left(g_{0}^{2}\right)=\left.b_{\mathrm{m}}\left(g_{0}^{2}\right)\right|_{\text {choice } 1}-\left.b_{\mathrm{m}}\left(g_{0}^{2}\right)\right|_{\text {choice } 2}$ in the right panel of figure 5 are found to be quite small, and their magnitude rapidly decreases as $a / L_{0} \rightarrow 0$.

\section{Outlook}

Apart from the elements sketched at the end of Section 2, which partly are already in progress, the computation of the b-quark mass at the $1 / m$-order of HQET along our strategy illustrated in figure 1 still requires $N_{\mathrm{f}}=2$ simulations in $L_{2} \approx 1 \mathrm{fm}$ as well as in physically large volumes of about $L_{\infty} \gtrsim 2 \mathrm{fm}$. Particularly for the latter we plan to switch to QCD with periodic boundary conditions and to use the technique of low-mode deflation [39] in connection with all-to-all quark propagators [40] for the numerical evaluation of correlation functions.

As further interesting directions for future work let us mention the non-perturbative tests of the HQET expansion in the spirit of ref. [22] and the extension of our determination of improvement coefficients and $Z$-factors to the parameter range relevant for (large volume) charm physics.

\section{Acknowledgments}

We thank NIC for allocating computer time on the APE computers to this project and the APE group at Zeuthen for its support. We further acknowledge partial support by the Deutsche Forschungsgemeinschaft (DFG) in the SFB/TR 09-03, "Computational Particle Physics", and by the European Community through EU Contract No. MRTN-CT-2006-035482, "FLAVIAnet". P.F. and J.H. also acknowledge financial support by the DFG under grant HE 4517/2-1.

\section{References}

[1] LHCb Collaboration, S. Barsuk, The LHCb experiment: Status and expected physics performance, Nucl. Phys. Proc. Suppl. 156 (2006) 93.

[2] SuperKEKB Physics Working Group Collaboration, A. G. Akeroyd et al., Physics at Super B Factory, hep-ex/0406071.

[3] E. Eichten, Heavy quarks on the lattice, Nucl. Phys. Proc. Suppl. 4 (1988) 170.

[4] E. Eichten and B. Hill, An effective field theory for the calculation of matrix elements involving heavy quarks, Phys. Lett. B234 (1990) 511. 
[5] ALPHA Collaboration, M. Della Morte, S. Dürr, J. Heitger, H. Molke, J. Rolf, A. Shindler and R. Sommer, Lattice HQET with exponentially improved statistical precision, Phys. Lett. $\mathbf{B 5 8 1}$ (2004) 93 [hep-lat/ 0307021 ]. Erratum: ibid. B612 (2005) 313.

[6] ALPHA Collaboration, M. Della Morte, A. Shindler and R. Sommer, On lattice actions for static quarks, J. High Energy Phys. 08 (2005) 051 [hep-lat/ 050600 8].

[7] ALPHA Collaboration, D. Guazzini, H. B. Meyer and R. Sommer, Non-perturbative renormalization of the chromo-magnetic operator in Heavy Quark Effective Theory and the $B^{*}-B$ mass splitting, arXiv:0705.1809 [hep-lat].

[8] F. Palombi, M. Papinutto, C. Pena and H. Wittig, Non-perturbative renormalization of static-light four-fermion operators in quenched lattice QCD, arXiv:0706.4153 [hep-lat] .

[9] ALPHA Collaboration, M. Della Morte, S. Dürr, D. Guazzini, J. Heitger, A. Jüttner and R. Sommer, Heavy-strange meson decay constants in the continuum limit of quenched $Q C D$, in preparation.

[10] ALPHA Collaboration, M. Della Morte, P. Fritzsch and J. Heitger, Non-perturbative renormalization of the static axial current in two-flavour QCD, J. High Energy Phys. 02 (2007) 079 [hep-lat/0611036].

[11] L. Maiani, G. Martinelli and C. T. Sachrajda, Nonperturbative subtractions in the heavy quark effective field theory, Nucl. Phys. B368 (1992) 281.

[12] E. Eichten and B. Hill, Static effective field theory: 1/m corrections, Phys. Lett. B243 (1990) 427.

[13] G. Martinelli and C. T. Sachrajda, Computation of the b-quark mass with perturbative matching at the next-to-next-to-leading order, Nucl. Phys. B559 (1999) 429 [hep-lat/9812001].

[14] F. D. Renzo and L. Scorzato, The residual mass in lattice heavy quark effective theory to $\alpha^{3}$ order, $J$. High Energy Phys. 02 (2001) 020 [hep-lat/ 0012011$].$

[15] ALPHA Collaboration, J. Heitger and R. Sommer, Non-perturbative heavy quark effective theory, J. High Energy Phys. 02 (2004) 022 [hep-lat/ 0310035].

[16] ALPHA Collaboration, M. Della Morte, N. Garron, M. Papinutto and R. Sommer, Heavy quark effective theory computation of the mass of the bottom quark, J. High Energy Phys. 01 (2007) 007 [hep-ph/0609294].

[17] R. Sommer, Non-perturbative QCD: renormalization, $O($ a)-improvement and matching to Heavy Quark Effective Theory, Lectures given at ILFTN Workshop on "Perspectives in Lattice QCD", Nara, Japan, 31 October - 11 November 2005 [hep-lat/0611020].

[18] M. Della Morte, SM parameters and heavy quarks on the lattice, PoS LAT2007 (2007) 008.

[19] ALPHA Collaboration, B. Blossier, M. Della Morte, N. Garron and R. Sommer, Heavy-light decay constant at the 1/m order of HQET, PoS LAT2007 (2007) 245.

[20] ALPHA Collaboration, M. Della Morte, R. Hoffmann, F. Knechtli, J. Rolf, R. Sommer, I. Wetzorke and U. Wolff, Non-perturbative quark mass renormalization in two-flavor QCD, Nucl. Phys. $\mathbf{B 7 2 9}$ (2005) 117 [hep-lat/0507035].

[21] ALPHA Collaboration, J. Heitger and J. Wennekers, Effective heavy-light meson energies in small-volume quenched QCD, J. High Energy Phys. 02 (2004) 064 [hep-lat/ 0312016 ].

[22] ALPHA Collaboration, J. Heitger, A. Jüttner, R. Sommer and J. Wennekers, Non-perturbative tests of heavy quark effective theory, J. High Energy Phys. 11 (2004) 048 [hep-ph / 0407227 ]. 
[23] M. Lüscher, R. Narayanan, P. Weisz and U. Wolff, The Schrödinger functional: A renormalizable probe for non-abelian gauge theories, Nucl. Phys. B384 (1992) 168 [hep-lat/920 0009 ].

[24] S. Sint, On the Schrödinger functional in QCD, Nucl. Phys. B421 (1994) 135 [hep-lat/9312079].

[25] ALPHA Collaboration, M. Kurth and R. Sommer, Renormalization and $O(a)$ improvement of the static axial current, Nucl. Phys. $\mathbf{B 5 9 7}$ (2001) 488 [hep-lat/ 0007002 ].

[26] ALPHA Collaboration, M. Della Morte, R. Frezzotti, J. Heitger, J. Rolf, R. Sommer and U. Wolff, Computation of the strong coupling in QCD with two dynamical flavours, Nucl. Phys. $\mathbf{B 7 1 3}$ (2005) 378 [hep-lat/0411025].

[27] ALPHA Collaboration, M. Guagnelli, R. Petronzio, J. Rolf, S. Sint, R. Sommer and U. Wolff, Non-perturbative results for the coefficients $b_{m}$ and $b_{A}-b_{P}$ in $O(a)$ improved lattice $Q C D$, Nucl. Phys. B595 (2001) 44 [hep-lat/ 0009021$].$

[28] ALPHA Collaboration, M. Della Morte, R. Hoffmann, F. Knechtli, R. Sommer and U. Wolff, Non-perturbative renormalization of the axial current with dynamical Wilson fermions, J. High Energy Phys. 07 (2005) 007 [hep-lat/ 0505026 ].

[29] ALPHA Collaboration, M. Della Morte, P. Fritzsch, B. Leder, H. Meyer, H. Simma, R. Sommer, $\mathrm{S}$. Takeda, $\mathrm{O}$. Witzel and U. Wolff, Preparing for $N_{\mathrm{f}}=2$ simulations at small lattice spacings, PoS LAT2007 (2007) 255.

[30] S. Duane, A. D. Kennedy, B. J. Pendleton and D. Roweth, Hybrid Monte Carlo, Phys. Lett. B195 (1987) 216.

[31] ALPHA Collaboration, H. B. Meyer, H. Simma, R. Sommer, M. Della Morte, O. Witzel and $\mathrm{U}$. Wolff, Exploring the HMC trajectory-length dependence of autocorrelation times in lattice $Q C D$, Comput. Phys. Commun. 176 (2007) 91 [hep-lat/ 0606004 ].

[32] J. C. Sexton and D. H. Weingarten, Hamiltonian evolution for the hybrid Monte Carlo algorithm, Nucl. Phys. B380 (1992) 665.

[33] C. Urbach, K. Jansen, A. Shindler and U. Wenger, HMC algorithm with multiple time scale integration and mass preconditioning, Comput. Phys. Commun. 174 (2006) 87 [hep-lat/0506011].

[34] M. Hasenbusch, Speeding up the Hybrid-Monte-Carlo algorithm for dynamical fermions, Phys. Lett. B519 (2001) 177 [hep-lat / 0107019 ].

[35] M. Hasenbusch and K. Jansen, Speeding up lattice QCD simulations with clover-improved Wilson fermions, Nucl. Phys. B659 (2003) 299 [hep-lat/ 0211042].

[36] ALPHA Collaboration, M. Della Morte, F. Knechtli, J. Rolf, R. Sommer, I. Wetzorke and U. Wolff, Simulating the Schrödinger functional with two pseudofermions, Comput. Phys. Commun. 156 (2003) 62 [hep-lat/0307008].

[37] ALPHA Collaboration, U. Wolff, Monte Carlo errors with less errors, Comput. Phys. Commun. 156 (2004) 143 [hep-lat/0306017].

[38] ALPHA Collaboration, S. Sint and P. Weisz, Further results on $O($ a) improved lattice $Q C D$ to one-loop order of perturbation theory, Nucl. Phys. B502 (1997) 251 [hep-lat/9704001].

[39] M. Lüscher, Local coherence and deflation of the low quark modes in lattice QCD, J. High Energy Phys. 07 (2007) 081 [arXiv: 0706.2298 [hep-lat] ].

[40] J. Foley, K. J. Juge, A. O'Cais, M. Peardon, S. M. Ryan and J. Skullerud, Practical all-to-all propagators for lattice QCD, Comput. Phys. Commun. 172 (2005) 145 [hep-lat/ 0505023 ]. 